\title{
Inactivation of Campylobacter jejuni using Radio-frequency Atmospheric Pressure Plasma on Agar Plates and Chicken Hams
}

\author{
Joo-Sung Kim, Eun-Jung Lee, Eun-Ah Cho, and Yun-Ji Kim* \\ Korea Food Research Institute, Seongnam 463-746, Korea
}

\begin{abstract}
Radio-frequency driven atmospheric pressure plasma using argon gas was studied in the inactivation of Campylobacter jejuni in order to investigate its applicability. First, the inactivation study was conducted on an agar surface. C. jejuni NCTC11168 was reduced by more than 7 Log CFU after an 88 s treatment. Another strain, ATCC49943, was studied; however, the inactivation was less efficient, with a 5 Log CFU reduction after a 2 min treatment. Then, chicken breast ham was studied at the $10^{6} \mathrm{CFU}$ inoculation level. The inactivation efficiency was much lower for both strains compared to that on the agar plates. C. jejuni NCTC11168 and ATCC49943 were reduced by 3 Log CFU after a 6 min treatment and by 1.5 Log CFU after a 10 min treatment, respectively. The scanning electron microscopy analysis indicated that $C$. jejuni cells were deformed or transformed into coccoid form under the plasma treatment. During the plasma treatment, the temperature of the samples did not rise above $43^{\circ} \mathrm{C}$, suggesting that heat did not contribute to the inactivation. Meanwhile, water activity significantly decreased after a $10 \mathrm{~min}$ treatment $(p<0.05)$. This study conveyed that radio-frequency atmospheric pressure plasma can effectively inactivate $C$. jejuni with strain-specific variation.
\end{abstract}

Key words: Campylobacter jejuni, radio-frequency atmospheric pressure plasma, inactivation, chicken breast ham, scanning electron microscopy

\section{Introduction}

Recently, interest in the non-thermal inactivation of microorganisms causing food-borne illnesses has increased. Non-thermal sterilization has an advantage over traditional thermal sterilization in that it can inactivate pathogens without deteriorating the sensory qualities of the food such as taste and flavor. Many non-thermal applications such as pulsed electric fields and high hydrostatic pressures have been studied and developed to satisfy the high demands for fresh foods (Senorans et al., 2003). In the same context, interest in non-thermal plasma technology has recently increased to study the feasibility of this technique for the inactivation of food-borne pathogenic bacteria.

A plasma is an ionized gas, the 'fourth state of matter'. As energy increases, the state of the material changes from solid to liquid, then to gas, and then to plasma. Gas such as argon or oxygen can be ionized using electrical energy and the ionization produces ionized gas, electrons,

*Corresponding author: Yun-Ji Kim, Korea Food Research Institute, Seongnam 463-746, Korea. Tel: 82-31-780-9085, Fax: 8231-780-9076, E-mail: yunji@kfri.re.kr neutrons, free radicals, and UV (Tendero et al., 2006; von Keudell et al., 2010). Plasma, especially the ions, can break chemical bonds as well as etch and damage biomolecules by the so-called 'chemical sputtering' (Moreau et al., 2008; von Keudell et al., 2010). Its oxidizing power and UV can also contribute to the damage process. Plasma has many applications such as wound healing, surface modification, and the sterilization of heat-sensitive medical instruments (Laroussi, 2005). It has been recently demonstrated that plasma can effectively inactivate various food-borne pathogenic bacteria such as Escherichia coli O157:H7, Listeria monocytogenes, Campylobacter, Salmonella, and Staphylococcus aureus (Critzer et al., 2007; Dirks et al., 2012; Niemira and Sites, 2008; Sureshkumar et al., 2010; Yun et al., 2010). For example, E. coli O157:H7 was reduced by 5 Log CFU and 3 Log CFU on agar plates and apples, respectively, after a 1-2 min treatment using a one atmosphere uniform glow discharge plasma (Critzer et al., 2007). Radio-frequency (RF) plasma reduced $S$. aureus by $6 \mathrm{Log}$ CFU using a $\mathrm{N}_{2}-\mathrm{O}_{2}$ mixture (Sureshkumar et al., 2010).

Campylobacter jejuni is one of the major food-borne pathogenic bacteria worldwide (Scallan et al., 2011; Young and Mansfield, 2005). This species is highly associated 
with poultry such as chicken, turkey, and duck (Humphrey et al., 2007). It causes diarrhea, abdominal cramps, and fever in humans even though most patients recover from the symptoms without any treatment (Young and Mansfield, 2005). Non-thermal dielectric barrier discharge plasma has been shown to reduce $C$. jejuni and $S$. enterica on the surface of chicken breast and thigh by 1-3 Log CFU (Dirks et al., 2012). In this study, we examined the ability of radio-frequency (RF) non-thermal plasma to inactivate $C$. jejuni on agar plates and chicken breast hams.

\section{Materials and Methods}

\section{C. jejuni strains, growth conditions, and prepara- tion of inocula}

C. jejuni NCTC11168 and ATCC49943 purchased from the American Type Culture Collection (USA) were used in this study. Bacterial strains in glycerol stocks at $-80^{\circ} \mathrm{C}$ were retrieved and grown on tryptic soy agar supplemented with $5 \%(\mathrm{v} / \mathrm{v})$ sheep blood (TSAB) at $37^{\circ} \mathrm{C}$ under microaerobic conditions $\left(6-12 \%(\mathrm{v} / \mathrm{v}) \mathrm{O}_{2}, 5-8 \%(\mathrm{v} / \mathrm{v}) \mathrm{CO}_{2}\right)$ generated using AnaeroPack-MicroAero (Mitsubishi Gas Chemical Co., Japan). After $32-48 \mathrm{~h}$ of incubation, the cultures were suspended in tryptic soy broth at an optical density (OD) of 0.1-0.2 at $600 \mathrm{~nm}$. An aliquot $(100 \mu \mathrm{L})$ of each culture was spread on TSAB plates and grown for an additional $14-15 \mathrm{~h}$ under the same conditions. The culture was suspended in tryptic soy broth at OD 0.1-0.2 $\left(10^{8}-10^{9} \mathrm{CFU} / \mathrm{mL}\right)$ using cell scrapers and then used as an inoculum.

\section{Plasma apparatus and treatment conditions}

An RF-driven atmospheric pressure plasma unit was used in this study (Fig. 1). This unit has an in-line plasma treatment system, and the length of plasma discharge is $160 \mathrm{~mm}$. Argon was used as a gas for plasma generation at a fixed flow rate of $20,000 \mathrm{sccm}$ with $200 \mathrm{~W}$ power. The argon gas was flushed into a horizontal electrode at which the plasma was generated. The plasma generated was then pushed downward in ambient air. The apparatus was equipped with a sample pedestal $(15 \mathrm{~cm} \times 15 \mathrm{~cm})$ on which samples were placed for exposure to the plasma treatment. The distance between the plasma discharge and the samples was approximately $2.0-2.5 \mathrm{~cm}$. A cooling pump was operated at $15^{\circ} \mathrm{C}$ to circulate a coolant to prevent the discharge unit from being overheated. For uniform plasma exposure, the sample pedestal moved back and forth in a range of $6.0 \mathrm{~cm}$ at $1.5 \mathrm{~cm} / \mathrm{s}$ in operation.

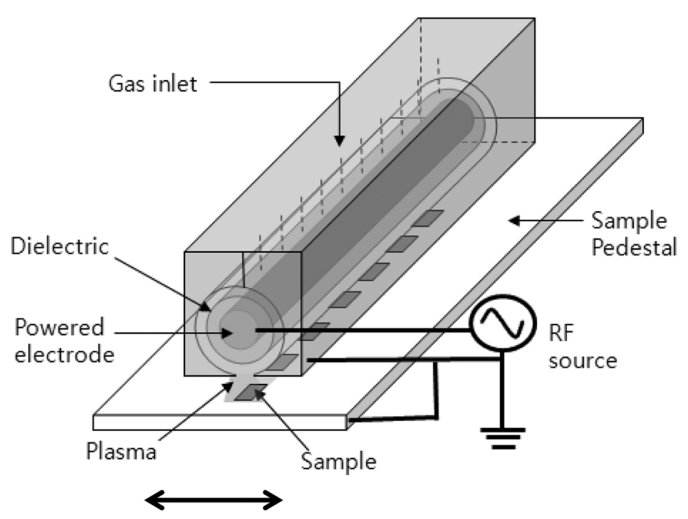

Fig. 1. Diagram of radio-frequency driven atmospheric pressure plasma used in this study.

\section{Plasma treatment on the agar plates}

Aliquots $(100 \mu \mathrm{L})$ of the prepared inoculum were gently spread $\left(10^{7}-10^{8} \mathrm{CFU}\right)$ with a pipette tip on plate count agar (BD, USA) in a $3.5 \mathrm{~cm}$ diameter circle. Then, the plates were semi-dried $(15 \mathrm{~min}-1 \mathrm{~h})$ in air. The plates were exposed to the plasma treatment for $0 \mathrm{~s}, 12 \mathrm{~s}, 24 \mathrm{~s}$, $40 \mathrm{~s}, 1 \mathrm{~min}, 1 \mathrm{~min} 28 \mathrm{~s}, 2 \mathrm{~min}$, or $5 \mathrm{~min}$ in duplicates. After the plasma treatment of the samples, the bacterial cells were suspended in $10 \mathrm{~mL}$ of sterile $0.85 \% \mathrm{NaCl}$ solution from the agar plates using cell scrapers, serially diluted in $0.85 \% \mathrm{NaCl}$ solution by 10 -fold to $10^{-5}$, and then the suspension and each dilution were spread on TSAB plates. The plates were incubated at $37^{\circ} \mathrm{C}$ under microaerobic conditions for approximately $48 \mathrm{~h}$, and the colonies grown on the plates were enumerated to determine the number of viable cells. By this method, around $100 \%$ of the viable cells were recovered from the agar plates.

\section{Plasma treatment on the chicken breast hams}

Chicken breast sliced hams ( $1 \mathrm{~mm}$ thick) were purchased from the markets and cut into $1 \mathrm{~cm} \times 1 \mathrm{~cm}$ pieces. The ham samples were placed on small petri dishes and inoculated with aliquots $(10 \mu \mathrm{L})$ of the inoculum at $10^{6}-10^{7}$ CFU. The inoculum was gently spread over the surface of the ham samples with a pipette tip. After incubation at room temperature for approximately $5 \mathrm{~min}$, the samples were exposed to the plasma treatment for $2 \mathrm{~min}, 4 \mathrm{~min}, 6$ $\mathrm{min}, 8 \mathrm{~min}$, or $10 \mathrm{~min}$ in duplicates. After the plasma treatment, the samples were vigorously agitated in $5 \mathrm{~mL}$ of sterile $0.85 \% \mathrm{NaCl}$ solution by vortex for $1 \mathrm{~min}$. Then, the supernatants were serially diluted in $0.85 \% \mathrm{NaCl}$ solution by 10 -fold to $10^{-5}$. The supernatants and each dilution were spread on TSAB supplemented with cefoperazone (32 $\mu \mathrm{g} / \mathrm{mL})$, vancomycin $(10 \mu \mathrm{g} / \mathrm{mL})$, and amphotericin B $(10 \mu \mathrm{g} / \mathrm{mL})$ for the selective growth of Campylobacter. The 
plates were incubated at $37^{\circ} \mathrm{C}$ under microaerobic conditions for 64-72 $\mathrm{h}$, and the colonies grown on the plates were enumerated. A negative control group of ham samples without any inoculation yielded no colonies on the plates.

\section{Scanning electron microscopy (SEM) pictures}

To prepare samples for SEM, C. jejuni cells on agar plates or on chicken breast hams were treated with plasma for $2 \mathrm{~min}$. The cells on agar plates were suspended in phosphate-buffered saline and pelleted by centrifugation. The pelleted cells from the agar plates or the cells on the chicken breast hams were fixed in a $2.5 \%$ paraformaldehyde-glutaraldehyde mixture buffered with $0.1 \mathrm{M}$ phosphate ( $\mathrm{pH}$ 7.2) for $2 \mathrm{~h}$. Then, the samples were post fixed in $1 \%$ osmium tetroxide in the same buffer for $1 \mathrm{~h}$, dehydrated in graded ethanol, substituted by isoamyl acetate, and dried at the critical point in $\mathrm{CO}_{2}$. Finally, the samples were sputtered with gold in a sputter coater (SC502, Polaron) and observed using the scanning electron microscope, HITACHI S4300N (HITACHI, Japan), installed at the Korea Research Institute of Bioscience \& Biotechnology (KRIBB).

\section{Temperature monitoring of the agar plates and chi- cken breast hams under plasma treatment}

To monitor the temperature on the agar plates and the chicken breast hams during plasma treatment, the temperature probe of a thermometer (50S K/J thermometer, Fluke, USA) was fixed onto the surface of the agar plates or stuck into the chicken breast sliced hams placed on the petri dish from the side. During the plasma treatment, the temperatures of the agar plate and the chicken breast hams were recorded every $8 \mathrm{~s}$ for $5 \mathrm{~min}$ and for $10 \mathrm{~min}$, respectively.

\section{Measurement of the water activity and water con-} tent of the ham samples

A previous method (AOAC, 2005) was followed to measure the water activity and the water content. To simulate the plasma treatment conditions, $10 \mu \mathrm{L}$ of tryptic soy broth was added to the chicken breast ham samples. After the plasma treatment, the water content was analyzed by drying samples at $105^{\circ} \mathrm{C}$ in a drying oven for $4 \mathrm{~h}$. The water content $(\%, w / w)$ was calculated as the ratio of weight loss after drying to the initial weight of the samples before drying. The water activity was measured by an Aw analyzer (ms1 Set aw, Novasina, Switzerland).

\section{Statistical analysis}

Differences in CFU between any two different treatment groups were analyzed by a t-test using Microsoft Office Excel 2007. P values less than 0.05 were considered significant. The data of the water activity and the water content were subjected to analysis of variance (ANOVA) using a SAS statistical program (SAS Institute Inc., USA). Multiple comparisons of the treatment groups were performed using Duncan's multiple range test for any significant differences.

\section{Results}

To determine if RF atmospheric pressure plasma can effectively inactivate $C$. jejuni, the plasma treatment was first tested on agar plates at an initial CFU of $10^{7}-10^{8}$ (Fig. 2). The CFU of $C$. jejuni NCTC11168 was significantly reduced by approximately $2 \log$ CFU $(p<0.05)$

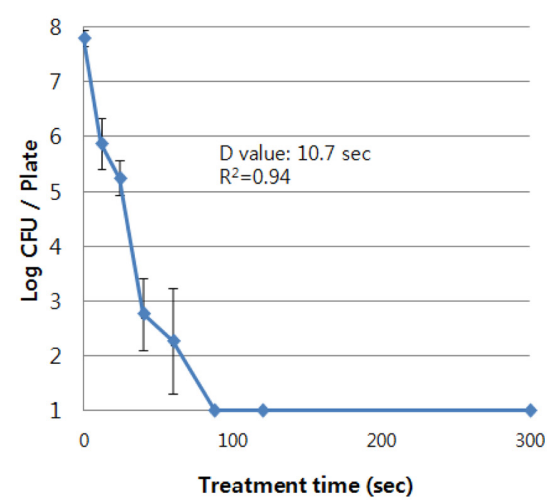

A

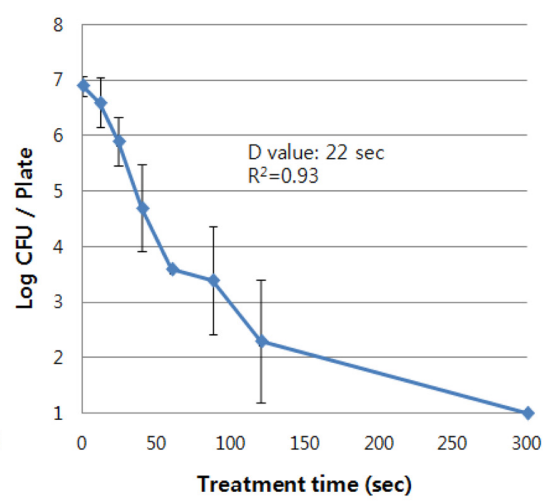

B

Fig. 2. Inactivation of (A) C. jejuni NCTC11168 and (B) $C$. jejuni ATCC49943 on agar plates with RF atmospheric pressure plasma. Data presented are based on 2-3 independent experiments. Detection limit was 1 Log CFU and any data below the detection limit were plotted at $1 \mathrm{Log}$ CFU. Error bar represents standard deviation. Decimal reduction time (D value) was calculated based on dynamic range of inactivation ( $0-60 \mathrm{~s}$ for NCTC11168; 0-88 s for ATCC49943). $\mathrm{R}^{2}$ represents correlation coefficient. 


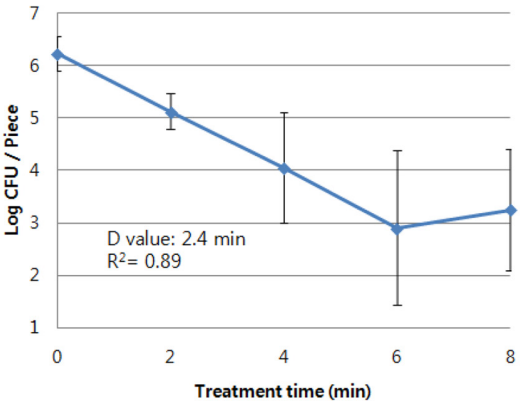

A

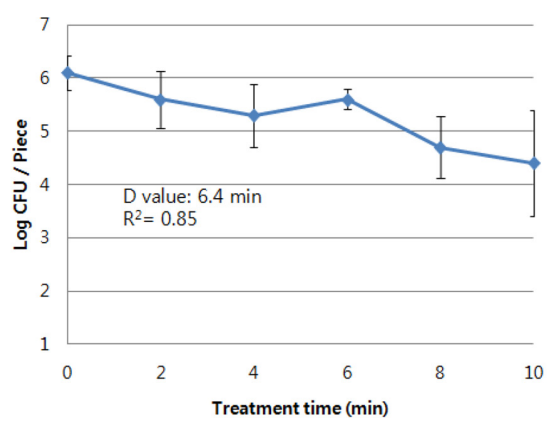

B

Fig. 3. Inactivation of (A) C. jejuni NCTC11168 and (B) C. jejuni ATCC49943 on chicken breast ham samples with RF atmospheric pressure plasma. Data presented are based on 2 independent experiments. Error bar represents standard deviation. $R^{2}$ represents correlation coefficient.

after an initial $12 \mathrm{~s}$ treatment (Fig. 2A). The inactivation continued with time and the $\mathrm{CFU}$ level fell below the detection limit after an $88 \mathrm{~s}$ treatment. Based on the inactivation kinetics, the decimal reduction time (D value) of the NCTC11168 strain was calculated as $10.7 \mathrm{~s}$. To study if any strain-specific variation exists in inactivation, another $C$. jejuni strain, ATCC49943, was studied under the same conditions. In this strain, significant reduction $(p<$ 0.05 ) occurred at $24 \mathrm{~s}$ instead of at $12 \mathrm{~s}$ (Fig. 2B). Although inactivation continued with time, inactivation was less efficient for ATCC49943 compared to that for NCTC11168 (Fig. 2). CFU counts of ATCC49943 were significantly higher than those of NCTC11168 at 12, 24, 40, 88, and $120 \mathrm{~s}(p<0.05)$ and the CFU level was still above the detection limit at the $2 \mathrm{~min}$ time point. Viable cells were non-detectable only at the $5 \mathrm{~min}$. Overall, the inactivation slope of strain ATCC49943 was not as steep as that of strain NCTC11168 and its D value was $22 \mathrm{~s}$ in contrast to the D value of $11 \mathrm{~s}$ of NCTC11168.

Because plasma treatment effectively inactivated both C. jejuni strains on agar plates, chicken breast ham samples purchased from markets were studied to evaluate inactivation efficacy (Fig. 3). The inactivation rate was much slower and less efficient on the ham samples compared to that on the agar plates. With an initial inoculation level at $10^{6}-10^{7} \mathrm{CFU}$, the plasma treatment reduced NCTC11168 by 1 Log CFU every 2 min until the $6 \mathrm{~min}$ time point, resulting in a $3 \mathrm{Log}$ reduction overall (Fig. $3 \mathrm{~A})$. Another $2 \mathrm{~min}$ treatment did not further inactivate the strain, suggesting a tailing effect. In the ham samples, inactivation was also less efficient for ATCC49943 than for NCTC11168, similar to the agar plates. Only a $1.5 \mathrm{Log}$ reduction occurred after an 8-10 min treatment of ATCC 49943 (Fig. 3B). The D values of NCTC11168 and ATCC 49943 in the ham samples were $2.4 \mathrm{~min}$ and $6.4 \mathrm{~min}$, res- pectively. These D values are approximately $10-20$ fold longer than those for the agar plates.

SEM analysis was conducted on C. jejuni NCTC11168 cells before and after the plasma treatment to study if the plasma treatment can affect the morphology of $C$. jejuni cells (Fig. 4). The C. jejuni cells had a typical spiral shape on both the agar plates (Fig. 4A) and the ham samples (Fig. 4C) before the plasma treatment. After the plasma treatment, however, its typical spiral shape was lost in many of the cells. They were deformed or transformed into a coccoid form in many of the cells on both the agar plates (Fig. 4B) and the ham samples (Fig. 4D). Despite the deformation, membrane damage was not apparent after the plasma treatment.

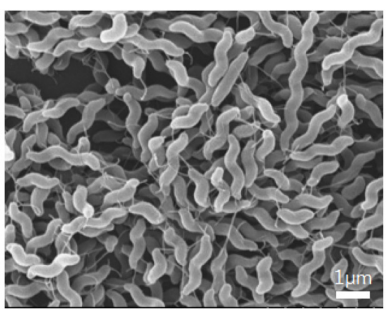

A

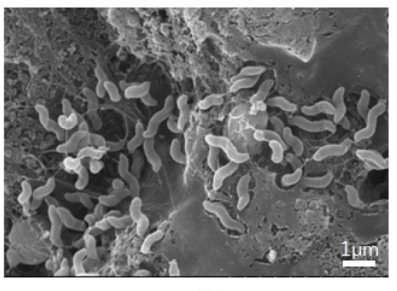

C

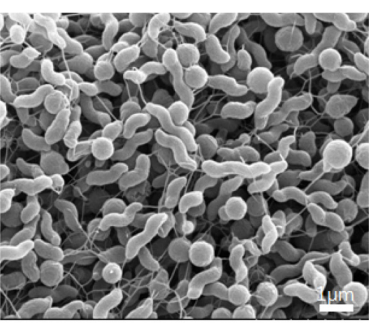

B

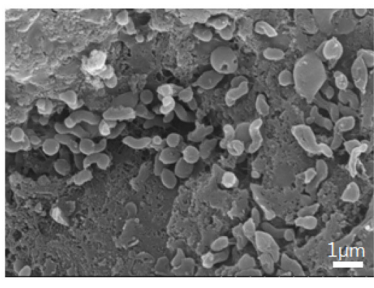

D
Fig. 4. SEM pictures of $C$. jejuni NCTC11168 either before or after plasma treatment on agar plates and chicken breast hams: (A) Before plasma treatment on agar plate, (B) After plasma treatment on agar plate, (C) Before plasma treatment on chicken breast ham, (D) After plasma treatment on chicken breast ham. Scale bar was inserted at right bottom corner. 


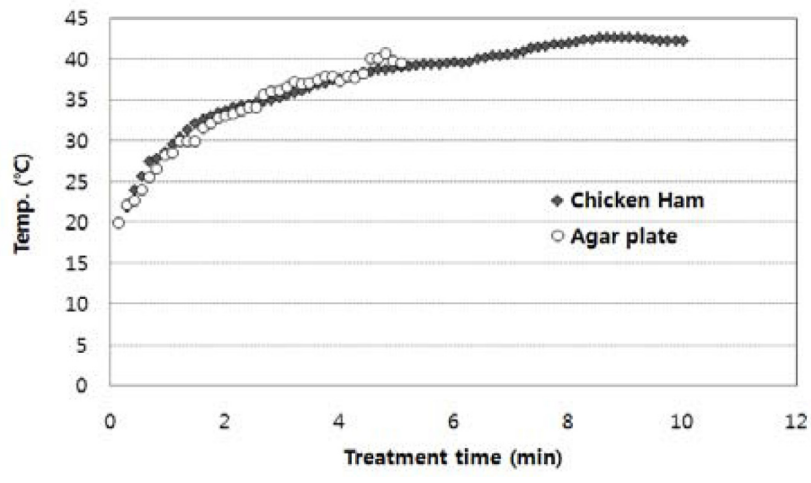

Fig. 5. Temperature monitoring of agar plates and chicken breast hams under plasma treatment. Temperature was recorded every $8 \mathrm{~s}$ in agar plates and chicken breast hams for $5 \mathrm{~min}$ and $10 \mathrm{~min}$, respectively.

Temperature was monitored on the agar plates and the ham samples during the plasma treatment to confirm that the treatment is non-thermal (Fig. 5). The plasma treatment was not completely non-thermal, and the temperature steadily increased for the first $2 \mathrm{~min}$. Then, it started to level off and did not further increase. The agar and ham samples reached only $41-43^{\circ} \mathrm{C}$ during the plasma treatment, suggesting that the inactivation of the $C$. jejuni cells was not related to the heat generated from the plasma.

It is known that plasma treatment has a desiccation effect (von Keudell et al., 2010). Therefore, the water activity and water content of the ham samples were measured to determine if our plasma treatment had any desiccation effect on the ham samples. The water activity was constant with a range of 0.934-0.935 at $0,2,4$, or 6 min of treatment with no statistically significant difference $(p>$ 0.05 ) among those treatment groups. However, the $8 \mathrm{~min}$ and $10 \mathrm{~min}$ treatment significantly decreased the water activity to 0.929 and 0.919 , respectively $(p<0.05)$. On the other hand, the water content gradually decreased as the treatment time increased. The water content significantly decreased from $67.9 \%$ to $65.3 \%$ after the 4 min treatment and to $59.6 \%$ after the $10 \mathrm{~min}$ treatment $(p<0.05)$. These data suggest that the plasma treatment had a desiccation effect on the ham samples.

\section{Discussion}

Although interest in plasma application to inactivate food-borne pathogenic bacteria has grown in recent years, the information regarding the effect of plasma treatment on $C$. jejuni is still very limited. In one recent study (Dirks et al., 2012), the $C$. jejuni contamination level was reduced by 2-3 Log CFU on chicken breast and thigh samples using a dielectric barrier discharge plasma after a 2-3 min treatment, which seems to be better than the performance of the RF plasma (0.5-1.5 Log reduction after the same time period) used in our study. However, it is still very difficult to draw conclusions because many of the conditions were different between the two studies such as the samples (raw chicken breast and thigh vs. chicken breast ham), initial inoculum levels $\left(10^{3}-10^{4} \mathrm{CFU}\right.$ vs. $10^{6}$ $10^{7} \mathrm{CFU}$ ), and the $C$. jejuni strains used (RM2002 vs. NCTC11168 and ATCC49943).

Such a large difference in the sterilization efficiency between the agar plates and the ham samples suggests that the sample surface condition is a critical factor in determining the sterilization efficiency of a plasma treatment. For example, Perni et al. (2008b) studied the inactivation of E. coli and L. monocytogenes and found that the inactivation of the pathogens on the cut surfaces of mango and cantaloupe was much slower than that on the membrane filters using a cold atmospheric plasma. When the pericarp was compared to the cut surface in mangos, the inactivation efficiency for $E$. coli was much lower on the cut surface compared to that on the pericarp (Perni et al., 2008a, b). The authors found that the bacterial cells had migrated into the interior through the cut surface, suggesting that the migrated cells inside the mango tissue could be protected from the plasma treatment. In our study, a population of $C$. jejuni cells could have migrated into the crevices in the ham samples and the plasma treatment might not have reached the population. Our hypothesis is supported by the rough surface and the crevices of the ham samples, as demonstrated in the SEM analysis (Fig. 4). The possible migration of the $C$. jejuni cells may explain the tailing phenomenon observed for NCTC11168 on the ham samples. A tailing phenomenon was not observed for the agar plates that have a flat surface without crevices. It was previously reported by Critzer et al. (2007) and Fernández et al. (2013) who studied Salmonella Enteritidis and Salmonella Typhimurium, respectively, on fresh produce such as cantaloupe, lettuce, strawberry, and potato of irregular surfaces compared to on agar or membrane filters of flat surfaces.

Based on the SEM analysis, the plasma treatment affected the cell morphology of the $C$. jejuni cells, changing their form from a typical spiral form to a coccoid form. The coccoid form in C. jejuni is often associated with its degeneration resulting from unfavorable conditions or environments such as aged culture, exposure to air, and heat or cold stresses (Buck et al., 1983; He and Chen, 2010; Jang et al., 2007; Moran and Upton, 1987b; Park, 2002; 
Tangwatcharin et al., 2006). Superoxide dismutase, a superoxide anion scavenging enzyme, can minimize the conversion of a spiral form to a coccoid form in air (Moran and Upton, 1987a). It suggests that reactive oxygen species can be involved in the conversion to a coccoid form. In this study, reactive oxygen species such as $\mathrm{OH}$ radicals may have been produced on the wet sample surface using the argon gas under atmospheric pressure in the plasma treatment (Srivastava and Wang, 2011). If so, these species could have played a role in the conversion of the spiral form to the coccoid form. However, cells with the coccoid form are not necessarily dead in C. jejuni (He and Chen, 2010). It suggests that it is still unclear if those coccoidal cells are dead in the plasma treatment. Aside from the conversion to coccoid form, there was no apparent damage on the cell membranes of $C$. jejuni in the SEM analysis. This result is in contrast to the results from other bacteria showing severe membrane damages, such as $E$. coli, Lactobacillus acidophilus, Streptococcus mutans (Korachi et al., 2010; Yang et al., 2011). Such a damaged cell membrane usually causes DNA or protein leakage (Deng et al., 2010; Yang et al., 2011). We attempted to detect DNA and protein leakage by measuring the absorbance of the supernatant at $260 \mathrm{~nm}$ and $280 \mathrm{~nm}$, respectively, after spinning down the suspended cells following the plasma treatment. However, we could not detect any significant increase in the DNA or protein concentrations in the plasma treatment group compared to those of the control group (data not shown), suggesting that no significant amount of DNA or protein leaked from C. jejuni cells after the plasma treatment. This result corresponds to the absence of obvious damage on the cell membranes of $C$. jejuni as observed in the SEM pictures.

The reason for a significant difference in the sensitivity to the plasma treatment between NCTC11168 and ATCC 49943 remains elusive. A previous study suggests that the surface morphology in a mold, Aspergillus niger, may affect the sensitivity to plasma treatment (von Keudell et al., 2010). Genetic diversity in C. jejuni strains is high, and extensive variation exists in the surface structures such as capsules and lipooligosaccharides (Dorrell et al., 2001; Parker et al., 2006; Wareing et al., 2002). Thus, variation in the surface structure may exist between NCTC11168 and ATCC49943. Not surprisingly, the genome of ATCC 49943 does not appear to be quite similar to that of NCTC 11168 (Hofreuter et al., 2006; Hofreuter et al., 2008).

Our data clearly show that the RF atmospheric pressure plasma has a desiccation effect on food samples. However, in this study, it should not have contributed to the inactivation of $C$. jejuni because the water activity did not significantly change up to the 6 or 8 min treatment. In addition, regarding plasma treatment on agar surface, no signs of desiccation were observed for at least 1 min treatment. It also suggests that desiccation does not account for most of the inactivation on the agar surface.

Nevertheless, this study still suggests that the desiccation effect by prolonged plasma treatment of meat samples may be undesirable because the decreases of water activity and water content could result in the increase of hardness on texture parameters (Jin et al., 2011; Serra et al., 2005). More studies are necessary to understand the effect of non-thermal plasma treatment on the sensory qualities of food products because few studies have been performed to our knowledge. In addition to the desiccation effect, there are several other potential limitations to overcome in the application of plasma for meat samples. For example, plasma can produce reactive oxygen species and its possible negative effects, such as lipid oxidation and off flavor, have yet to be studied. Other product quality issues such as discoloration have to be carefully studied and managed for industrial application. In fact, Rød et al. (2012) reported that the lipid oxidation occurred in ready-to-eat meat after plasma treatment even though it was still within the acceptable range for sensory qualities. According to Kim et al. (2011), a significantly higher level of lipid oxidation was observed in plasma-treated bacons compared to controls after $7 \mathrm{~d}$ storage. These studies suggest that the lipid oxidation may be an issue for plasma treatment of meat products and warrants for further studies. Overall, this study shows that non-thermal RF atmospheric pressure plasma has a potential application in inactivating $C$. jejuni on food samples, but additional studies are necessary to evaluate the effects of non-thermal plasma technology on the qualities of meat products.

\section{Acknowledgements}

This research was funded by Korea Food Research Institute. We thank FEMTO Science Co. for providing an RF atmospheric pressure plasma equipment.

\section{References}

1. AOAC. (2005) Official methods of analysis of the Association of Analytical Chemistry. Association of Official Analytical Chemists, Washington, DC, USA.

2. Buck, G. E., Parshall, K. A., and Davis, C. P. (1983) Electron microscopy of the coccoid form of Campylobacter jejuni. J. Clin. Microbiol. 18, 420-421. 
3. Critzer, F. J., Kelly-Wintenberg, K., South, S. L., and Golden, D. A. (2007) Atmospheric plasma inactivation of foodborne pathogens on fresh produce surfaces. J. Food Prot. 70, 22902296.

4. Deng, S., Cheng, C., Ni, G., Meng, Y., and Chen, H. (2010) Bacillus subtilis devitalization mechanism of atmosphere pressure plasma jet. Curr. Appl. Phys. 10, 1164-1168.

5. Dirks, B. P., Dobrynin, D., Fridman, G., Mukhin, Y., Fridman, A., and Quinlan, J. J. (2012) Treatment of raw poultry with nonthermal dielectric barrier discharge plasma to reduce Campylobacter jejuni and Salmonella enterica. J. Food Prot. 75, 22-28.

6. Dorrell, N., Mangan, J. A., Laing, K. G., Hinds, J., Linton, D., Al-Ghusein, H., Barrell, B. G., Parkhill, J., Stoker, N. G., Karlyshev, A. V., Butcher, P. D., and Wren, B. W. (2001) Whole genome comparison of Campylobacter jejuni human isolates using a low-cost microarray reveals extensive genetic diversity. Genome Res. 11, 1706-1715.

7. Fernández, A., Noriega, E., and Thompson, A. (2013) Inactivation of Salmonella enterica serovar Typhimurium on fresh produce by cold atmospheric gas plasma technology. Food Microbiol. 33, 24-29.

8. He, Y. and Chen, C. Y. (2010) Quantitative analysis of viable, stressed and dead cells of Campylobacter jejuni strain 81176. Food Microbiol. 27, 439-446.

9. Hofreuter, D., Novik, V., and Galan, J. E. (2008) Metabolic diversity in Campylobacter jejuni enhances specific tissue colonization. Cell Host Microbe. 4, 425-433.

10. Hofreuter, D., Tsai, J., Watson, R. O., Novik, V., Altman, B., Benitez, M., Clark, C., Perbost, C., Jarvie, T., Du, L., and Galan, J. E. (2006) Unique features of a highly pathogenic Campylobacter jejuni strain. Infect. Immun. 74, 4694-4707.

11. Humphrey, T., O’Brien, S., and Madsen, M. (2007) Campylobacters as zoonotic pathogens: a food production perspective. Int. J. Food Microbiol. 117, 237-257.

12. Jang, K. I., Kim, M. G., Ha, S. D., Kim, K. S., Lee, K. H., Chung, D. H., Kim, C. H., and Kim, K. Y. (2007) Morphology and adhesion of Campylobacter jejuni to chicken skin under varying conditions. J. Microbiol. Biotechnol. 17, 202-206.

13. Jin, S.-K., Shin, D., and Hur, I.-C. (2011) Effects of moisture content on quality characteristics of dry-cured ham during storage. Korean J. Food Sci. An. 31, 756-762.

14. Kim, B., Yun, H., Jung, S., Jung, Y., Jung, H., Choe, W., and Jo, C. (2011) Effect of atmospheric pressure plasma on inactivation of pathogens inoculated onto bacon using two different gas compositions. Food Microbiol. 28, 9-13.

15. Korachi, M., Gurol, C., and Aslan, N. (2010) Atmospheric plasma discharge sterilization effects on whole cell fatty acid profiles of Escherichia coli and Staphylococcus aureus. J. Electrostatics 68, 508-512.

16. Laroussi, M. (2005) Low temperature plasma-based sterilization: overview and state-of-the-art. Plasma Process Polym. 2, 391-400

17. Moran, A. P. and Upton, M. E. (1987a) Effect of medium supplements, illumination and superoxide dismutase on the production of coccoid forms of Campylobacter jejuni ATCC
29428. J. Appl. Bacteriol. 62, 43-51.

18. Moran, A. P. and Upton, M. E. (1987b) Factors affecting production of coccoid forms by Campylobacter jejuni on solid media during incubation. J. Appl. Bacteriol. 62, 527-537.

19. Moreau, M., Orange, N., and Feuilloley, M. G. (2008) Nonthermal plasma technologies: New tools for bio-decontamination. Biotechnol. Adv. 26, 610-617.

20. Niemira, B. A. and Sites, J. (2008) Cold plasma inactivates Salmonella Stanley and Escherichia coli O157:H7 inoculated on golden delicious apples. J. Food Prot. 71, 1357-1365.

21. Park, S. F. (2002) The physiology of Campylobacter species and its relevance to their role as foodborne pathogens. Int. J. Food Microbiol. 74, 177-188.

22. Parker, C. T., Quinones, B., Miller, W. G., Horn, S. T., and Mandrell, R. E. (2006) Comparative genomic analysis of Campylobacter jejuni strains reveals diversity due to genomic elements similar to those present in C. jejuni strain RM1221. J. Clin. Microbiol. 44, 4125-4135.

23. Perni, S., Liu, D. W., Shama, G., and Kong, M. G. (2008a) Cold atmospheric plasma decontamination of the pericarps of fruit. J. Food Prot. 71, 302-308.

24. Perni, S., Shama, G., and Kong, M. G. (2008b) Cold atmospheric plasma disinfection of cut fruit surfaces contaminated with migrating microorganisms. J. Food Prot. 71, 1619-1625.

25. Rød, S. K., Hansen, F., Leipold, F., and Knøchel, S. (2012) Cold atmospheric pressure plasma treatment of ready-to-eat meat: Inactivation of Listeria innocua and changes in product quality. Food Microbiol. 30, 233-238.

26. Scallan, E., Hoekstra, R. M., Angulo, F. J., Tauxe, R. V., Widdowson, M. A., Roy, S. L., Jones, J. L., and Griffin, P. M. (2011) Foodborne illness acquired in the United States--major pathogens. Emerg. Infect. Dis. 17, 7-15.

27. Senorans, J., Ibanez, E., and Cifuentes, A. (2003) New trends in food processing. Crit. Rev. Food Sci. Nutr. 43, 507-526.

28. Serra, X., Ruiz-Ramírez, J., Arnau, J., and Gou, P. (2005) Texture parameters of dry-cured ham $m$. biceps femoris samples dried at different levels as a function of water activity and water content. Meat Sci. 69, 249-254.

29. Srivastava, N., and Wang, C. (2011) Effects of water addition on $\mathrm{OH}$ radical generation and plasma properties in an atmospheric argon microwave plasma jet. J. Appl. Phys. 110, 053304.

30. Sureshkumar, A., Sankar, R., Mandal, M., and Neogi, S. (2010) Effective bacterial inactivation using low temperature radio frequency plasma. Int. J. Pharm. 396, 17-22.

31. Tangwatcharin, P., Chanthachum, S., Khopaibool, P., and Griffiths, M. W. (2006) Morphological and physiological responses of Campylobacter jejuni to stress. J. Food Prot. 69, 27472753.

32. Tendero, C., Tixier, C., Tristant, P., Desmaison, J., and Leprince, P. (2006) Atmospheric pressure plasmas: A review. Spectrochimica Acta Part B. 61, 2-30.

33. von Keudell, A., Awakowicz, P., Benedikt, J., Raballand, V., Yanguas-Gil, A., Opretzka, J., Flötgen, C., Reuter, R., Byelykh, L., Halfmann, H., Stapelmann, K., Denis, B., Wunderlich, J., Muranyi, P., Rossi, F., Kylián, O., Hasiwa, N., Ruiz, A., Rauscher, H., Sirghi, L., Comoy, E., Dehen, C., Challier, L., and 
Deslys, J. P. (2010) Inactivation of bacteria and biomolecules by low-pressure plasma discharges. Plasma Process. Polym. 7, 327-352.

34. Wareing, D. R., Bolton, F. J., Fox, A. J., Wright, P. A., and Greenway, D. L. (2002) Phenotypic diversity of Campylobacter isolates from sporadic cases of human enteritis in the UK. J. Appl. Microbiol. 92, 502-509.

35. Yang, B., Chen, J., Yu, Q., Li, H., Lin, M., Mustapha, A., Hong, L., and Wang, Y. (2011) Oral bacterial deactivation using a lowtemperature atmospheric argon plasma brush. J. Dent. 39, 48-56.
36. Young, V. B. and Mansfield, L. S. (2005) Campylobacter infection-clinical context. In: Campylobacter molecular and cellular biology. Ketley, J. M. and Konkel, M. E. (ed) Horizon bioscience, UK, pp. 1-12.

37. Yun, H., Kim, B., Jung, S., Kruk, Z. A., Kim, D. B., Choe, W., and Jo, C. (2010) Inactivation of Listeria monocytogenes inoculated on disposable plastic tray, aluminum foil, and paper cup by atmospheric pressure plasma. Food Control 21, 11821186.

(Received 2012.11.26/Revised 2013.4.2/Accepted 2013.4.28) 